\title{
Chapter 18 \\ The Relation of ERP Indices of Exercise to Brain Health and Cognition
}

\author{
Charles H. Hillman, Keita Kamijo, and Matthew B. Pontifex
}

\begin{abstract}
This chapter describes a program of research aimed at the relation of physical activity to brain health and cognition, with implications for scholastic achievement among youth. We describe a body of neurophysiological research that indicates that both chronic and acute physical activity relate to enhanced cognitive function, albeit over a different temporal duration. Such findings have important implications for public health in school age children, as physical activity stands to increase brain health and cognitive function during childhood and across the life span.
\end{abstract}

\section{C.H. Hillman $(\bowtie)$}

Department of Kinesiology and Community Health, University of Illinois at Urbana-Champaign, 317 Louise Freer Hall; 906 S. Goodwin Avenue, Champaign, IL, USA

Department of Psychology, University of Illinois at Urbana-Champaign, 317 Louise Freer Hall; 906 South Goodwin Avenue, Urbana, IL 61801, USA

Department of Internal Medicine, University of Illinois at Urbana-Champaign, 317 Louise Freer Hall; 906 South Goodwin Avenue, Urbana, IL 61801, USA

The Beckman Institute for Advanced Science and Technology, University of Illinois at Urbana-Champaign, 317 Louise Freer Hall; 906 South Goodwin Avenue, Urbana, IL 61801, USA

Division of Neuroscience, University of Illinois at Urbana-Champaign, 317 Louise Freer Hall; 906 South Goodwin Avenue, Urbana, IL 61801, USA

Division of Nutritional Sciences, University of Illinois at Urbana-Champaign, 317 Louise Freer Hall; 906 South Goodwin Avenue, Urbana, IL 61801, USA

e-mail: chhillma@illinois.edu

K. Kamijo $\bullet$ M.B. Pontifex

Department of Kinesiology and Community Health, University of Illinois at Urbana-Champaign, 316 Louise Freer Hall; 906 S. Goodwin Avenue, Champaign, IL, USA

e-mail: k-kamijo@aoni.waseda.jp; pontifex@msu.edu 


\subsection{Introduction}

There is a growing public health burden of inactivity in industrialized nations. In recent years, children have become increasingly sedentary, leading to concomitant increases in the prevalence of being overweight and unfit (DHHS and DOE 2000). Despite the knowledge that physical activity has broad benefits for public health, including increased physical fitness, maintenance of healthy body weight, and a reduced risk of disease (Strong et al. 2005), greater than one third of children do not meet the national guidelines of moderate to vigorous activity on a daily basis (CDC 2008). During adulthood, even fewer individuals meet national guidelines for physical activity (Haskell et al. 2007). At issue, physical inactivity during early childhood has implications for physical activity behaviors across the life span, and has been related to the prevalence of several chronic diseases (e.g., cardiovascular disease and type-2 diabetes) during adolescence and adulthood. In fact, recent estimates have suggested that younger generations in the United States may lead shorter and less healthy lives than their parents, marking the first time in U.S. history that a trend such as this has occurred (Fontaine et al. 2003; Olshansky et al. 2005). These estimates are based on the rise in overweight and obesity status and the fall of physical activity behaviors among children.

However, absent from these public health concerns is the relation of physical inactivity on brain health and cognition. Not too far into our history, our early ancestors engaged in a much more rigorously active lifestyle, suggesting that our evolutionary past may have been shaped by an active lifestyle for much of human existence (Bortz II 1985; Fialkowski 1986). Specifically, it has been conjectured that human evolution (including brain) was shaped during our formative years as hunters (Bortz II 1985). Such a lifestyle required strenuous physical activity through the prolonged, persistent pursuit of prey under considerable heat stress (Fialkowski 1986). As a function of this lifestyle, there is speculation that human beings became specialized for (among other behaviors) endurance physical activity (Carrier 1984), with additional supposition that rapid increases in brain volume occurred to meet the demands of physically active hunting behaviors under heat stress (Fialkowski 1986). Accordingly, brain and body evolved in concert to support the demands of considerable movement.

In many regards, it is unfortunate that contemporary human beings have, for the most part, removed the need for strenuous physical activity from daily life. Vaynman and Gomez-Pinilla (2006, p. 700) state "Ironically, in a world that recognizes the benefits of exercise, physical inactivity characterizes most industrialized societies of our modern age. This is likely due to the benefits reaped by technological advances, which have obviated many of the necessities for physical labor." This statement suggests that human beings may be maladapted to the sedentary lifestyle of today (Booth and Lees 2006; Vaynman and Gomez-Pinilla 2006), with evidence indicating not only poorer physical health but also poorer cognitive health, as suggested by an increasing scientific focus on the relation of physical activity to brain and cognition (see Hillman et al. 2008 for review). 
Thus, the purpose of this chapter is to describe a subset of the available data on the relation of physical activity to brain health and cognition across the human life span. In particular, a programmatic approach to this area of research has used measures, which tap the neuroelectric system to provide a greater understanding of cognitive functioning, beyond what may be understood through overt behavioral measures. Electroencephalography (EEG) is considered the oldest measure for assessing functional brain activation. Despite many new advances in neuroimaging, EEG remains a useful tool in understanding covert cognitive operations. The work described in this chapter focuses on the use of EEG to understand the relation of physical activity to brain health and cognition, with a particular focus on the event-related potential (ERP) approach. The overall purpose of this research model is to better understand factors that relate to cognitive health and effective functioning across the human life span. However, before turning to the review, we first describe the concept of cognitive control and briefly review ERPs to provide the necessary background for the literature reviewed. We then review the extant findings of exercise, brain health, and cognition, with a particular focus on ERP indices of cognitive control.

\subsection{Cognitive Control}

The term cognitive control (also referred to as "executive control") describes an overarching set of higher-order, cognitive operations, which are involved in the regulation of goal-directed interactions within the environment (Botvinick et al. 2001; Meyer and Kieras 1997; Norman and Shallice 1986). These processes allow for the optimization of behavior through the selection, scheduling, coordination, and maintenance of computational processes underlying aspects of perception, memory, and action (Botvinick et al. 2001; Meyer and Kieras 1997; Miyake et al. 2000; Norman and Shallice 1986). The core cognitive processes which are collectively termed "cognitive control" include inhibition, working memory, and cognitive flexibility (Diamond 2006). Although these processes are distinct and distinguishable, it is important to note that they share common neural structures and are functionally interrelated (Miyake et al. 2000). Inhibitory control often requires one to deliberately override a dominant response in order to perform a less potent but correct response, suppress task-irrelevant information in the stimulus environment, or stop an ongoing response (Barkley 1997; Davidson et al. 2006). This ability to inhibit attention to task-irrelevant or distracting stimuli is thought to be central to the ability to sustain attention and allow control over one's actions. That is, it allows one to make decisions based on choice, rather than impulse. Working memory refers to the ability to hold information in one's mind and manipulate it and is heavily involved in each of the other cognitive control processes as it also relates to the ability to represent internal goals and standards for the comparison of those goals against current performance in order to regulate behavior (Bunge and Crone 2009). Lastly, cognitive 
flexibility relates to the ability to hold multiple goals, response mappings, and internal representations within the mind and adaptively switch perspectives and attentional foci to meet environmental demands (Diamond 2006). It is these processes, which have demonstrated protracted development during maturation, and early decay during aging, which are among some of the most affected by physical activity behaviors.

\subsection{Event-Related Brain Potentials}

Beyond the assessment of overt actions, ERPs provide a means of gaining insight into the relationship between physical activity/fitness and cognitive control through the examination of a subset of neurophysiological processes that occur between stimulus encoding and response production. Accordingly, these measures allow for a more precise understanding of the relation of exercise-induced changes on cognition. ERPs refer to a class of neuroelectric activity that occurs in response to, or in preparation for, a stimulus or response (Coles et al. 1990). This neuroelectric activity is reflective of the synchronous activity of large populations of neurons (Hugdahl 1995), and can reflect obligatory responses (exogenous) or higher-order cognitive processing that often require active participation from the individual (endogenous; Hugdahl 1995). The stimulus-locked ERP is characterized by a succession of positive $(\mathrm{P})$ and negative $(\mathrm{N})$ components, which are constructed according to their direction and the relative time that they occur (Hruby and Marsalek 2003). Earlier components (N1, P2) of the stimulus-locked potential relate to aspects of selective attention, while later components (N2, P3) relate to various aspects of endogenous cognitive function (e.g., response inhibition, attentional resource allocation; see Fig. 18.1).

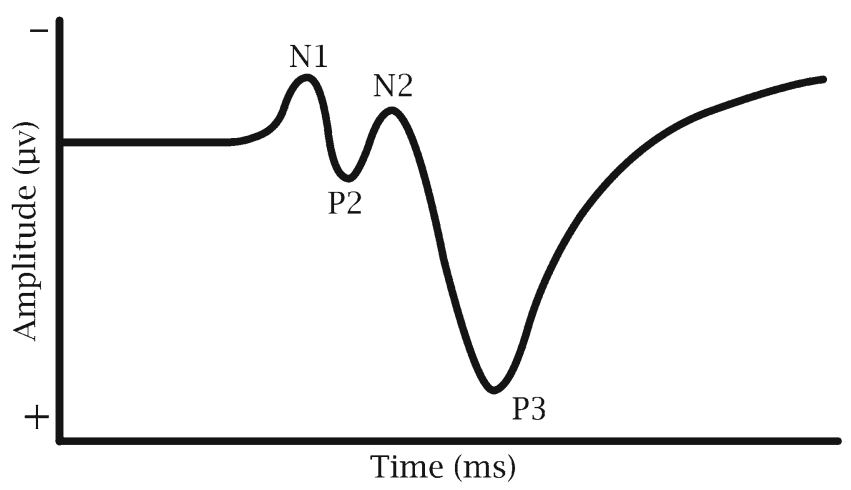

Fig. 18.1 Characterization of a stimulus-locked event-related potential denoting the N1, P2, N2, and $\mathrm{P} 3$ components 


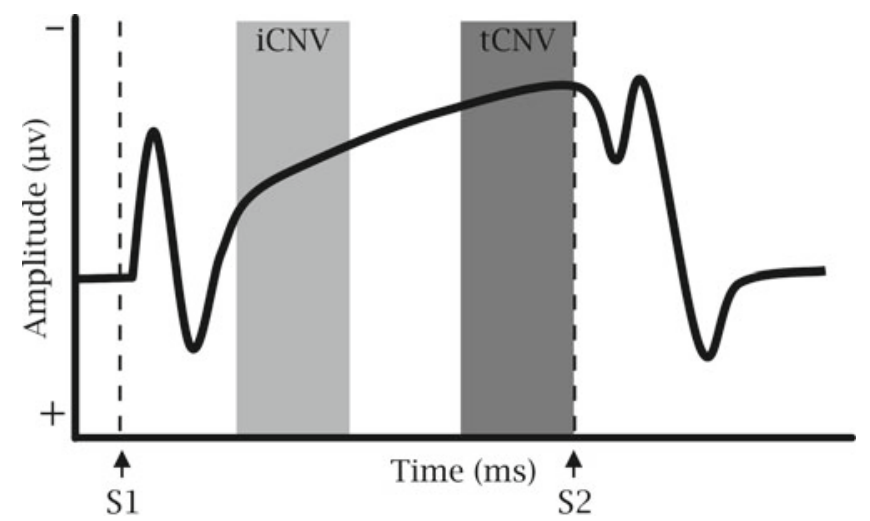

Fig. 18.2 Characterization of a stimulus-locked event-related potential denoting the initial CNV (iCNV) and the terminal $\mathrm{CNV}(\mathrm{tCNV})$ and their relationship with warning (S1) and imperative (S2) stimuli

Among ERP components, the P3 (also known as the P300 or P3b) has garnered considerable attention in the literature in regard to the relation of physical activity/fitness to neurocognition. Originally discovered in 1965 by Sutton, Braren, Zubin, and John, the P3 is a positive-going deflection occurring approximately 300-800 ms after stimulus presentation, with a topographic maximum at electrode sites over the parietal cortex (Polich and Kok 1995). This endogenous component reflects neuronal activity associated with the revision of the mental representation of the previous event (Donchin 1981), such that the P3 is sensitive to the allocation of attentional resources during stimulus engagement (Polich 2007). Based on a recent theoretical account of the P3 by Polich (2007), the amplitude is believed to be proportional to the amount of resources allocated toward the suppression of extraneous neuronal activity in order to facilitate attentional processing, such that larger P3 amplitude would reflect greater inhibition of superfluous activity. P3 latency is generally considered to reflect stimulus detection and evaluation time (Ilan and Polich 1999; Magliero et al. 1984), which is independent of response selection and behavioral action (Verleger 1997). Further, P3 latency appears to be negatively correlated with mental function, with shorter latencies related to superior cognitive performance (Emmerson et al. 1989; Howard and Polich 1985; Johnson et al. 1985; Polich and Martin 1992; Polich et al. 1983). Although the precise neural origins of the P3 are still unknown, the generation of the $\mathrm{P} 3$ appears to result from the interaction between frontal and temporal/parietal networks with additional contributions stemming from a number of subcortical structures (Ebmeier et al. 1995; Kirino et al. 2000; Polich 2003).

A second ERP component of interest with regard to physical activity/fitness related changes in cognition is the contingent negative variation (CNV; see Fig. 18.2), a negative-going slow cortical potential elicited during the interval between warning (S1) and imperative (S2) stimuli. The term CNV more broadly refers to at least two different ERP components: the initial CNV (iCNV; also called O-wave) 


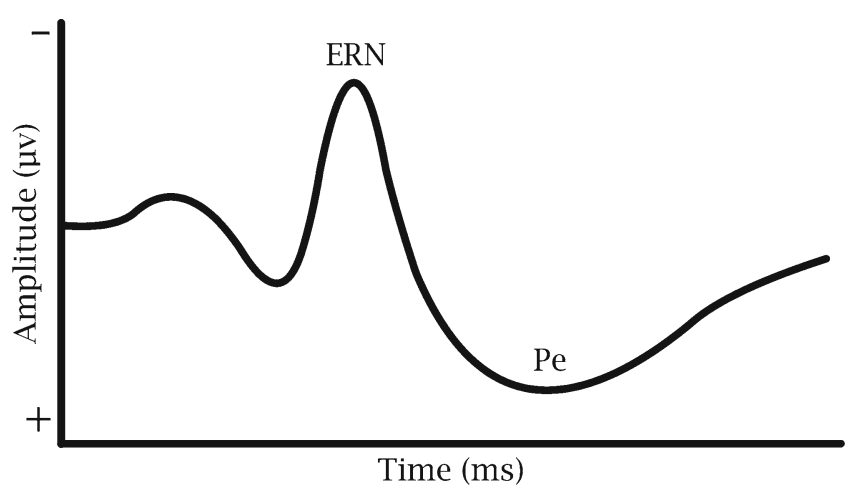

Fig. 18.3 Characterization of a response-locked event-related potential denoting the error-related negativity (ERN) and error positivity (Pe)

and the terminal CNV (tCNV; also called E-wave; Loveless and Sanford 1974; Weerts and Lang 1973). The iCNV has been associated with stimulus orientation with a frontal topographic maximum, while the tCNV is thought to relate to stimulus anticipation and/or response preparation and demonstrates a central topographic maximum (Brunia and van Boxtel 2001; Loveless and Sanford 1974; van Boxtel and Brunia 1994; Weerts and Lang 1973). Further, recent evidence suggests that a more frontal topographic distribution of the CNV is associated with cognitive preparatory processes rather than response preparation (Falkenstein et al. 2003; Leynes et al. 1998; Lorist et al. 2000; Wild-Wall et al. 2007). The CNV has multiple neural generators including the anterior cingulate cortex (ACC), supplementary motor area, prefrontal cortex, and primary motor cortex (Cui et al. 2000; Gómez et al. 2003; Hamano et al. 1997).

Lastly, a separate class of ERPs is time-locked to an individual's response (see Fig. 18.3). One such component of this "response-locked" ERP is the error-related negativity (ERN; also known as the Ne), which is described as a negative-going deflection occurring approximately 50-150 ms after errors of commission with a topographic maximum over fronto-central recording sites (Falkenstein et al. 1991; Gehring et al. 1993). The ERN is believed to reflect the activation of action monitoring processes in response to erroneous behaviors to initiate the upregulation of topdown compensatory processes to correct an individual's responses during subsequent environmental interaction (Falkenstein et al. 1991; Gehring and Knight 2000; Gehring et al. 1993). The ERN has been found to occur regardless of an individual's awareness of error commission (Nieuwenhuis et al. 2001). The neural tissue underlying the generation of the ERN has been localized through hemodynamic (Carter et al. 1998), magneto-encephalographic (Miltner et al. 2003), and high-density dipole modeling (Dehaene et al. 1994; van Veen and Carter 2002) studies to the dorsal portion of the ACC. Accordingly, through the measurement of these (and other) ERP components, information concerning the specific aspects of cognition that are influenced by exercise behaviors may be better understood. 


\subsection{The Relation of Chronic Physical Activity Participation, Aerobic Fitness, and ERPs}

Since Kramer et al. (1999) first reported that increases in aerobic fitness selectively improved performance on tasks requiring greater amounts of cognitive control, there has been growing interest in the selective effects of exercise on cognitive control over the past decade. A meta-analysis (Colcombe and Kramer 2003) supported the notion that although regular physical activity provides general benefits across multiple aspects of cognition, the improvements are selectively and disproportionately greater for processes requiring extensive amounts of cognitive control. However, it should be noted that researchers have not reached consensus on the relationship of chronic physical activity to cognition (see Smith et al. 2010 for review). Regardless, several ERP studies have focused on the general versus selective nature of the relationship between chronic physical activity and cognitive function across the life span. Specifically, these studies are classified into five categories based on types of cognitive task and cognitive processes examined: stimulus discrimination, inhibition, working memory, action monitoring, and cognitive flexibility.

\subsubsection{Stimulus Discrimination}

Early ERP studies examined the relation of physical activity and aerobic fitness to cognitive function using an oddball task (Dustman et al. 1990; Polich and Lardon 1997). The oddball task requires participants to respond to rare target stimuli (i.e., oddballs) among a train of frequent nontarget stimuli (e.g., 20\% target and 80\% nontarget). The oddball task requires relatively minimal cognitive control compared to other tasks as will hereafter be described. These pioneering studies indicated that chronic physical activity and aerobic fitness are positively associated with increased attentional resources during stimulus engagement (larger P3 amplitude) in young adults (Polich and Lardon 1997) and faster cognitive processing speed (i.e., shorter P3 latency) in older adults (Dustman et al. 1990). Thus, early findings provided evidence that chronic physical activity and aerobic fitness are positively related to stimulus discrimination processes in adult populations.

A more recent ERP study (Pontifex et al. 2009b) observed that greater aerobic fitness was associated with larger P3 amplitude during an oddball task in both young and older adults, replicating the earlier findings of Polich and Lardon (1997). However, Pontifex et al. 2009b) also manipulated perceptual discrimination difficulty of the oddball task, and indicated that greater aerobic fitness was associated with increased P3 amplitude irrespective of age for the easier task condition, whereas the positive relationship between fitness and P3 amplitude was only observed in young adults for the more difficult task condition. These findings suggested that fitness may not be sufficient to overcome age-related cognitive deficits when task difficulty is too great (Pontifex et al. 2009b). Thus, it would appear that the positive relation of aerobic 
fitness to cognitive function is mediated by age and task difficulty. The general relationship between fitness and cognitive function has been also observed in preadolescent children, as higher-fit children exhibited larger P3 amplitude and shorter P3 latency during the oddball task compared to their lower-fit counterparts (Hillman et al. 2005). Collectively, these ERP studies using variants of the oddball task suggest that chronic participation in physical activity and greater aerobic fitness are associated with superior stimulus discrimination across the life span.

\subsubsection{Inhibition}

Several ERP studies have used modifications of the Eriksen flanker task (Eriksen and Eriksen 1974) to investigate the relation of chronic physical activity and aerobic fitness to inhibitory aspects of cognitive control. This task requires participants to respond to a centrally presented target stimulus amid an array of flanking stimuli, which are task irrelevant. A typical flanker task consists of congruent trials, in which the target letter is flanked by the same letters (e.g., HHHHH or SSSSS), and incongruent trials, in which the target letter is flanked by opposing letters that are mapped to a different response (e.g., SSHSS or HHSHH). Incongruent trials require greater amounts of inhibitory control to gate out interference generated by the flanker stimuli due to the activation of the incorrect response mapping. Hillman et al. (2004) compared the P3 component of young adults with high, moderate, and low physically active older adults during a flanker task. Results indicated that high and moderate active older adults exhibited larger P3 amplitude compared to young adults only for the incongruent condition, while no such differences were observed for low active older adults. Given that larger P3 amplitude, reflecting greater allocation of attentional resources, was observed for both high and moderately active adults, the data suggest that a particular threshold of physical activity may be necessary to accrue neurocognitive benefits. Alternatively, young adults exhibited shorter P3 latency relative to the older adults, replicating the robust finding that cognitive processing speed slows during cognitive aging. However, when physical activity was considered among the older adults, greater physical activity levels were associated with shorter P3 latencies, suggesting a linear relationship between activity level and cognitive processing speed. Thus, these findings suggest that physical activity may be positively related to cognitive function during tasks requiring variable amounts of inhibitory control in older adults.

More recently, Hillman et al. (2009a) extended these findings in older adults to preadolescent children. Specifically, 9-10 year old higher aerobically fit children exhibited larger P3 amplitude and greater response accuracy compared to their lower aerobically fit counterparts during a flanker task, while no such fitness-related differences were observed for P3 latency. Accordingly, these data may indicate that during preadolescent development, fitness may provide a benefit to attention directed toward the stimulus environment during tasks requiring variable amounts of inhibitory control, but that processing speed is not influenced in the same manner. 
It is worth noting that other authors have reported no relation of fitness to P3 indices of cognition in adolescent children during a flanker task (Stroth et al. 2009). However, deeper inspection into the study design yields several possible explanations for this contradiction. First, Stroth et al. (2009) used a median split to bifurcate their sample into higher and lower aerobically fit groups. That is, moderate-fit participants might have been included in both groups, which may blur differences in fitness-related inhibitory function between groups. Second, Stroth et al. (2009) used a modified flanker/Go-NoGo task instead of a more traditional flanker task that has been incorporated into prior ERP studies (Hillman et al. 2004, 2009a; Pontifex et al. 2011). Specifically, the flanker/Go-NoGo task consisted of eight types of letter arrays (congruent: BBBBB, DDDDD, UUUUU, and VVVVV; incongruent: DDBDD, BBDBB, VVUVV, and UUVUU). Participants were asked to respond to centrally presented "B" and "U" letters, and to withhold their response to centrally presented "D" and "V" letters. As such, the hybrid flanker/Go-NoGo task necessitates greater working memory demands to maintain multiple rule sets, and flexibly allocate and inhibit these rule sets, compared to standard flanker tasks. As described above, the relation of fitness to cognitive function may be mediated by task difficulty (Pontifex et al. 2009b). Thus, the flanker/Go-NoGo task might have been too difficult for adolescent children to detect subtle differences in inhibitory function between groups (Stroth et al. 2009). Lastly, the P3 component was measured at electrode sites overlying the lateral portions of the central scalp region (Stroth et al. 2009). It is well established that P3 amplitude has a topographic maximal amplitude over the midline-parietal region (Polich 2007), and most ERP studies have indicated the positive relation of physical activity to the P3 component along midline electrode sites (e.g., Hillman et al. 2004; Kamijo and Takeda 2009; Polich and Lardon 1997). Thus, these methodological differences may have accounted for the contradictory findings between Stroth et al. (2009) and other ERP studies using the flanker task (Hillman et al. 2004, 2009a).

Finally, other inhibitory control tasks have been used to assess the relation of chronic physical activity to this aspect of cognition. Kamijo and Takeda (2009) observed a positive relation of physical activity to inhibitory control using a spatial priming task (Tipper et al. 1990) in young adults. In this task, a target letter ("O") and a distractor letter ("X") were presented at two of four possible locations simultaneously. Participants were required to respond to the target location and to ignore the distractor location. When the target in the current trial appeared at the distractor location in the previous trial, RTs become longer compared to other trials (Tipper et al. 1990). This effect, which has been termed "negative priming", is considered to reflect inhibitory control to prevent interference from distractors on working memory. In other words, a larger negative priming effect may reflect superior inhibitory control (Kamijo and Takeda 2009). The results indicated that physically active young adults exhibited larger negative priming effects on RT and P3 latency compared to their sedentary peers, suggesting that regular physical activity is associated with inhibitory control processes during young adulthood. Taken together, the majority of ERP studies that have used inhibition tasks to assess the relation of chronic physical activity and/or aerobic fitness to cognition have observed a 
positive relation of these variables to inhibitory control across the life span. Despite differences in tasks and age, the majority of studies report similar P3 findings, suggesting a robust relationship between chronic engagement of exercise and this aspect of cognition.

\subsubsection{Working Memory}

In addition to the body of research described above that has used stimulus discrimination tasks to examine the allocation of attentional resources, recent research in our laboratory supports a positive relation of physical activity to working memory using a Sternberg task in young adults (Kamijo et al. 2010). The Sternberg task requires participants to encode a memory set (S1) containing an array of letters (e.g., XFJTP) and respond whether a single probe (S2; e.g., ??T??) appeared in the encoded array (S1). In this task, greater working memory demands are required to encode and maintain relevant information for larger set sizes. Further, the Sternberg task allows for the measurement of task preparation processes between S1 and S2 with the CNV. Kamijo et al. (2010) conducted the Sternberg task under differential instructions to respond as accurately as possible or as quickly as possible to manipulate cognitive control demands, because greater allocation of resources is needed during task preparation processes requiring a speeded response (Falkenstein et al. 2003). Kamijo et al. (2010) reported significantly smaller frontal tCNV amplitude for higher aerobically fit compared to lower aerobically fit participants during the speed instruction, while no such relationship was observed during the accuracy instruction. From these findings it was proposed that higher aerobically fit individuals might maintain a more constant level of cognitive control irrespective of the task instructions, resulting in more efficient cognitive preparation (i.e., smaller frontal tCNV) during the speed condition compared to lower aerobically fit individuals (Kamijo et al. 2010). Thus, the CNV findings support a positive relation of chronic physical activity to working memory. Given that preliminary evidence suggests that preadolescent children may derive similar benefits to working memory as a result of increased aerobic fitness (Kamijo et al. 2011), it would appear that chronic physical activity may be positively associated with working memory irrespective of age.

\subsubsection{Action Monitoring}

Action monitoring is another important aspect of cognitive control. That is, to orchestrate goal-directed behaviors, individuals must monitor and correct response errors during environmental interaction for the maintenance and adaptation of successful task performance. Several studies have employed the ERN to examine the relation of chronic physical activity and aerobic fitness to action monitoring. 
Initially, two studies indicated that greater amounts of chronic physical activity participation (Themanson et al. 2006) and aerobic fitness (Themanson and Hillman 2006) were associated with smaller ERN amplitude and larger post-error response slowing in young and older adults under speeded task instructions. This relationship has subsequently been observed in preadolescent children, with higher aerobically fit children exhibiting smaller ERN amplitude and greater post-error response accuracy compared to lower aerobically fit children (Hillman et al. 2009a; Pontifex et al. 2011). Based on the conflict monitoring theory (Botvinick et al. 2001; Carter and van Veen 2007), the ACC, which is a neural generator of the ERN (Carter et al. 1998; Dehaene et al. 1994; Miltner et al. 2003; van Veen and Carter 2002), monitors response conflict and signals the dorsolateral prefrontal cortex to upregulate cognitive control in support of subsequent environmental interaction. As such, longer RT and greater accuracy on trials following errors are believed to reflect the upregulation of cognitive control via the adoption of a more conservative response set. Thus, smaller ERN amplitude for higher physically active and aerobically fit individuals may reflect a reduction in conflict or a lower threshold in which to begin the cascade of processes required for the upregulation of cognitive control (Hillman et al. 2009a; Pontifex et al. 2011; Themanson and Hillman 2006; Themanson et al. 2006).

Although it would appear that this interpretation is convenient on the surface, a functional magnetic resonance imaging (fMRI) study is consonant with the reduced ERN findings in higher aerobically fit individuals. Colcombe et al. (2004) investigated the relationship between aerobic fitness and brain activity using fMRI using both a cross-sectional assessment and a randomized clinical trial. They showed that higher-fit and aerobically trained older adults exhibited less activation in the ACC, along with greater activation of task-related prefrontal and parietal brain regions during a flanker task. In addition, higher-fit/aerobically trained participants performed better on the flanker task compared to the lower-fit/nonaerobic control older adults (Colcombe et al. 2004). These results imply that higher aerobically fit, relative to lower aerobically fit, individuals may interact within the stimulus environment using a strategy that is either less dependent on action monitoring or requiring less activation (i.e., reduced ACC activation) to upregulate cognitive control (i.e., increased prefrontal activation) for subsequent behavioral adaptation. Based on these data, it is plausible that the decreased ERN amplitude, which has been localized to the dorsal ACC, may reflect the same differences in cognitive strategy between higher-active/higher-fit individuals and their less active/fit counterparts (Hillman et al. 2009a; Pontifex et al. 2011; Themanson and Hillman 2006; Themanson et al. 2006). Collectively, greater levels of physical activity and higher amounts of aerobic fitness may be associated with increased cognitive control, resulting in a lower threshold for detection and signaling of conflict (decreased ERN) and greater response slowing to preserve response accuracy on subsequent trials. Thus, these ERN studies suggest that physical activity has a positive relation with the efficiency of the action monitoring system across the life span and support the positive relation of physical activity and aerobic fitness to cognitive control. 


\subsubsection{Cognitive Flexibility}

It is well established that ERN amplitude is increased when instructions stress response accuracy over response speed, which is thought to reflect the increased salience of the error (Gehring et al. 1993) or increased attentional focus due to the emphasis placed upon correct action (Yeung et al. 2004). That is, it would appear that the instruction to respond as accurately as possible requires greater regulation of cognitive control relative to the speed instruction during action monitoring processes. Themanson et al. (2008) examined the relationship between aerobic fitness and ERN in young adults with manipulation of task instructions (i.e., accuracy vs. speed instructions). Results showed that greater aerobic fitness was associated with larger ERN amplitude and greater post-error accuracy under the accuracy instruction, whereas no such association was observed under the speed instruction (Themanson et al. 2008). Interestingly, fitness was positively associated with greater modulation of ERN amplitude and post-error accuracy across the task instruction conditions (i.e., accuracy—speed condition; Themanson et al. 2008). These results suggest that greater aerobic fitness may be associated with increased cognitive flexibility of action monitoring during tasks requiring variable cognitive demands.

Pontifex et al. (2011) extended the findings of aerobic fitness to cognitive flexibility in preadolescent children through manipulation of stimulus-response compatibility using a modified flanker task. Specifically, in the compatible stimulus-response condition, participants were required to press a button consonant with the direction of the centrally presented target arrow, whereas in the incompatible stimulus-response condition, participants pressed a button that opposed the direction of the central target arrow. Prior research indicates that the incompatible, relative to the compatible, condition requires greater flexibility in the modulation of cognitive control (Friedman et al. 2009). Pontifex et al. (2011) found that higher aerobically fit children could preserve response accuracy across the compatibility conditions, whereas lower aerobically fit children exhibited decreased response accuracy for the incompatible compared to compatible condition. In addition, higher aerobically fit children exhibited larger P3 amplitude for the incompatible compared to the compatible condition, while no such a difference was observed for lower aerobically fit children, who exhibited overall smaller P3 responses (Pontifex et al. 2011).

Furthermore, higher aerobically fit children exhibited greater modulation of ERN amplitude based on the compatibility conditions, in a manner similar to prior research in adults (Themanson et al. 2008). Specifically, higher aerobically fit children had smaller ERN amplitude compared to lower aerobically fit children, suggesting a decrease in response conflict during environmental interaction. However, when manipulation of stimulus-response compatibility was considered, the fitnessERN relationship was observed for the compatible, but not the incompatible, condition. That is, higher aerobically fit children had reduced ERN amplitude in the compatible condition relative to their lower aerobically fit peers, replicating prior findings in children (Hillman et al. 2009a) and adults (Themanson et al. 2006). However, during the incompatible condition, which requires greater amounts of 
cognitive control, higher aerobically fit children had a significantly greater ERN potential that did not differ from lower aerobically fit participants, but differed significantly from the ERN response that they exhibited during the compatible condition. This pattern of results suggests that higher aerobically fit children have a greater capacity to flexibly modulate action monitoring processes based on cognitive control demands to optimize behavioral interactions within the task environment. The modulation of ERN across conditions with greater amounts of fitness is also consistent with the above-mentioned P3 amplitude findings (Pontifex et al. 2011). These findings imply that higher aerobically fit children may recruit more attentional resources during stimulus engagement and upregulate action monitoring processes to preserve response accuracy for the task condition requiring greater amounts of cognitive control (i.e., incompatible condition). Alternatively, lower aerobically fit children might not be able to modulate stimulus engagement and action monitoring processes based on cognitive control requirements, which may result in lower response accuracy for the incompatible condition. Taken together, these ERP studies (Pontifex et al. 2011; Themanson et al. 2008) suggest that fitness is positively associated with a greater capability to flexibly modulate cognitive control during stimulus engagement and action monitoring processes in adults and children.

Other research has pursued more traditional means of examining cognitive flexibility as a function of physical activity and fitness. That is, cognitive flexibility requirements have often been manipulated using task-switching paradigms, which require individuals to attend to stimuli in their environment, inhibit response tendencies, hold multiple rule sets in the contents of their working memory, and flexibily execute responses based on the various rule sets. A typical task-switching paradigm consists of homogeneous and heterogeneous task conditions. The homogeneous condition includes a single rule set (i.e., AAAAAA ... or BBBBBB...) and requires individuals to make a response based on the stimuli presented. The heterogeneous condition consists of two or more tasks (i.e., multiple rule sets), and requires the individual to flexibly switch between task rules across individual trials (e.g., $\mathrm{ABABAB} \ldots$ or AABBAA...). The heterogeneous condition results in longer RT and decreased accuracy compared to the homogeneous condition. The RT difference between homogeneous and heterogeneous conditions has been labeled the "global switch costs" (Bojko et al. 2004; Rogers and Monsell 1995), which is believed to reflect the demands of maintaining two or more rule sets active in working memory (Kray and Lindenberger 2000; Rogers and Monsell 1995).

Hillman et al. (2006) examined the relation of physical activity to working memory and mental flexibility using a task-switching paradigm in both young and older physically active and sedentary adults. Their results indicated that chronically active individuals had shorter P3 latency compared to sedentary individuals for the heterogeneous condition, but not for the homogeneous condition, across age groups. In other words, the relationship between chronic physical activity and cognitive processing speed was only observed for the task condition requiring greater working memory demands and mental flexibility. This finding suggests that physical activity is positively associated with the cognitive control of working memory during mental flexibility tasks. Further, 
Kamijo and Takeda (2010) indicated that chronic physically active young adults had smaller global switch costs compared to sedentary young adults. In addition, sedentary participants exhibited smaller P3 amplitude for the heterogeneous condition relative to the homogeneous condition, whereas such a difference in P3 amplitude was not observed for active individuals (Kamijo and Takeda 2010). Based on these findings, active individuals may be able to maintain attentional resources toward a stimulus during task conditions requiring variable working memory demands (i.e., the maintenance of working memory during the heterogeneous condition), which in turn may underlie the production of a smaller global switch cost.

Finally, it should be noted that other researchers have not observed the positive relation of aerobic fitness to cognitive performance using a task-switching paradigm (Scisco et al. 2008). One possible explanation for this discrepancy relates to differences in task difficulty among these ERP studies. That is, Hillman et al. (2006) used two tasks involving single digit numbers (digits 1-9, excluding 5), in which participants were asked to discern whether the number was greater or lesser than 5 (low/high task), or whether the number was odd or even (odd/even task). Alternatively, Scisco et al. (2008) used four tasks involving double digit numbers (digits 11-99), in which participants were required to judge whether numbers were greater or lesser than 50 , whether the numbers were odd or even, whether the sum of two digits was greater or lesser than 10 , or whether the sum of the two digits was odd or even. Obviously, task-switching paradigms including four tasks (Scisco et al. 2008) are more difficult compared to those involving only two tasks (Hillman et al. 2006), and as such there may be limits to working memory capacity and the ability to inhibit specific rule sets in favor of executing others, beyond which physical activity cannot influence cognitive performance.

Kamijo and Takeda (2010) analyzed the greater/lesser than task and odd/even tasks separately during the above-mentioned task-switching paradigm with two tasks, because task difficulty differed between the two tasks, as the greater/lesser than task exhibited shorter RT and greater accuracy (i.e., easier) compared to the odd/ even task. Further, they found a selective relation of chronic physical activity to RT and P3 amplitude as a function of cognitive control demands only for the greater/ lesser than task (Kamijo and Takeda 2010). From these findings, and coupled with the well-established findings that the P3 component is sensitive for task difficulty (McCarthy and Donchin 1981; Polich 1987; Pontifex et al. 2009a, b), it is plausible that task difficulty may interact with the relationship between chronic physical activity and neurocognitive performance during tasks requiring mental flexibility. Accordingly, the null relationship between aerobic fitness and cognitive performance in Scisco et al. (2008) may be due to the difficulty of their task-switching paradigm. Thus, the relation of chronic physical activity to task difficulty should be explored further to better elucidate the cognitive health benefits to mental flexibility.

\subsubsection{Summary}

Previous ERP studies reviewed herein used various types of cognitive tasks to explore the general and selective relationship between chronic physical activity and 
cognitive function based on the manipulation of cognitive control requirements. Overall, it would appear that greater participation in physical activity and higher amounts of aerobic fitness are associated with overall superior cognitive function. However, this overall positive relation appears selectively and disproportionately larger for tasks or task components requiring greater amounts of cognitive control. Lastly, although the dataset comprising the relation of physical activity/aerobic fitness to cognitive control is not extensive, the ERP studies reviewed herein have been, for the most part, consonant in suggesting a positive relation with the various aspects of cognitive control irrespective of age.

\subsection{The Acute Effects of Single Bouts of Physical Activity on ERPs}

Given the apparent link between chronic physical activity and cognition discussed above, a growing body of research has been focused on elucidating how a single acute bout of physical activity may serve to modulate cognitive processes. However, an important distinction must be made with regard to acute exercise investigations, which separate the time during which cognition is assessed. That is, to better understand the influence of a single acute bout of physical activity on cognition one must separate those investigations, which have assessed cognition during physical activity from those that have assessed cognition following physical activity participation.

\subsubsection{Neuroelectric Changes in Cognition Following a Single Acute Bout of Physical Activity}

Within the small body of research that has investigated the transient after-effects of a single acute bout of physical activity in adults, findings suggest a beneficial influence on a variety of cognitive functions (Brisswalter et al. 2002; Lambourne and Tomporowski 2010; Tomporowski 2003b), with a disproportionately larger benefit for tasks or task components requiring greater cognitive control demands (Hillman et al. 2003, 2009b; Pontifex et al. 2009a). These findings are consonant with previous research into the effects of acute exercise on cognition in children, with improvements in cognition observed for both simple and choice RT tasks (Ellemberg and St-Louis-Deschênes 2010), aspects of concentration (Caterino and Polack 1999; Mahar et al. 2006; McNaughten and Gabbard 1993), mathematics (Gabbard, and Barton 1979), brief tests of academic achievement (Hillman et al. 2009b), and inhibitory control (Hillman et al. 2009b) following participation in single acute bouts of structured physical activities lasting at least $20 \mathrm{~min}$. However, to date, only a handful of previous investigations have examined the aftereffects of a single acute bout of aerobic exercise on neuroelectric indices of cognition. 


\subsubsection{Stimulus Discrimination}

At first glance, investigations that have assessed the influence of single acute bouts of exercise on stimulus discrimination processes using oddball tasks appear to demonstrate conflicting findings. However, a clearer picture emerges when these findings are considered relative to the intensity of the aerobic exercise bout. Specifically, Kamijo et al. (2004) examined the extent to which the relative intensity of a single acute bout of exercise might differentially influence the P3 component in a sample of college-aged young adults performing a Go task (similar to an oddball task). Findings from this investigation revealed an increase in P3 amplitude only following an acute bout of moderate intensity aerobic exercise, while very light and very high intensity acute exercise did not modulate P3 amplitude (Kamijo et al. 2004). Similarly, Nakamura and colleagues (1999) observed increased P3 amplitude in response to an auditory oddball task following a 30 min acute bout of moderately intense exercise relative to baseline. However, investigations that have assessed the influence of high intensity and maximal intensity aerobic exercise on stimulus discrimination have largely observed no acute exercise-induced modulations of P3 amplitude (Duzova et al. 2005; Yagi et al. 1999), save for one noted exception (Magnié et al. 2000). That is, Magnié et al. (2000) examined a sample of collegeaged young adults at rest and following a maximal graded exercise test on a cycle ergometer in response to an auditory oddball task. Findings from this investigation revealed larger P3 amplitude and shorter P3 latency following a single acute bout of maximal exercise, in contrast to the findings of Duzova et al. 2005. It is unclear why these investigations demonstrated conflicting findings; however, given the limited body of research to date, future research will further elucidate the relationship of acute exercise intensity and stimulus discrimination. Despite the lack of consensus for high intensity exercise, the extant literature on single acute bouts of physical activity and stimulus discrimination indicates that moderate intensity exercise is beneficial to neuroelectric indices reflecting the allocation of attentional resources in the service of stimulus discrimination.

\subsubsection{Inhibition}

Of any cognitive process, inhibition has been the most studied in the acute exercise literature (e.g., Hillman et al. 2003, 2009b; Kamijo et al. 2004, 2007, 2009) and relates to the ability to act on the basis of choice rather than impulse (Davidson et al. 2006). The first investigation of the influence of acute exercise on neuroelectric indices of inhibition was conducted by Hillman et al. (2003), who assessed inhibitory control using a modified flanker task at rest and following a 30 min bout of aerobic exercise at an intensity of $80-85 \%$ of maximum heart rate in a sample of 20 college-aged young adults. Findings revealed that following exercise, participants exhibited an increased allocation of attentional resources as indexed by larger P3 
amplitude. Further, reduced interference was found for the task condition requiring greater amounts of cognitive control, as indexed by nonsignificant differences in P3 latency across the neutral and incongruent trials following exercise. This effect was not observed during rest, as the incongruent condition elicited longer P3 latency relative to the neutral condition. Accordingly, these findings were the first to demonstrate that single bouts of aerobic exercise had acute and transient effects on neuroelectric processes underlying inhibitory control through an increase in the allocation of neuroelectric resources and alterations in cognitive processing and stimulus classification speed (Hillman et al. 2003).

Building from this investigation Kamijo et al. (2007) assessed the extent to which the relative intensity of an acute aerobic exercise bout modulated these neuroelectric processes underlying inhibitory control. Specifically, Kamijo et al. (2007) examined neuroelectric changes in response to a modified flanker task in a sample of 12 college-aged young adults during a resting baseline session, and following light (at an rating of perceived exertion [RPE] of 11), moderate (at an RPE of 13), and hard (at an RPE of 15) cycling. Although no behavioral differences were observed, findings from this investigation revealed increased P3 amplitude following light and moderate aerobic exercise with no changes following hard exercise relative to the resting baseline condition (Kamijo et al. 2007). Further, replicating Hillman et al. (2003), Kamijo et al. (2007) observed selectively shorter P3 latencies for incongruent trials across light, moderate, and hard exercise conditions.

Additional support for the beneficial effects of acute exercise on neurocognitive processes has been garnered from other research employing a different inhibitory control task. Specifically, Kamijo et al. (2004) examined the influence of a single bout of aerobic exercise on the P3 component in college-aged young adults in response to a NoGo task following very light, moderate, and very hard exercise intensities. Findings revealed that larger P3 amplitude was observed for the NoGo condition, which requires aspects of response inhibition, following an acute bout of moderate intensity aerobic exercise, an effect that was not observed following very light or very hard intensity exercise. Accordingly, these findings (Hillman et al. 2003; Kamijo et al. 2004, 2007) indicate that in college-aged young adults, an increase in the allocation of attentional resources (i.e., larger P3 amplitude) occurs following single bouts of light to moderate intensity aerobic exercise, while facilitations of cognitive processing and stimulus classification speed occur following aerobic exercise of any intensity.

To date only two investigations have assessed neuroelectric indices of cognition in response to inhibitory control tasks in participant populations other than college-aged young adults. Kamijo et al. (2009) assessed the influence of acute aerobic exercise on cognition in a sample of 12 older adults relative to 12 collegeaged young adults. Participants completed the modified flanker task at rest and again following 20 min of light exercise at an intensity of 55\% of heart rate max, and moderate exercise at an intensity of $74 \%$ of heart rate max. Findings from this investigation replicated findings from Hillman et al. (2003) and Kamijo et al. (2007) with increased P3 amplitude following moderately intense exercise in young adults, while no such effect was observed for older adults. Interestingly, 
regardless of age and exercise intensity shorter P3 latency was observed following exercise relative to rest. Accordingly, these findings suggest that mechanisms underlying the effects of acute exercise on the allocation of attentional resources may be age-dependent, while cognitive processing speed is enhanced by short duration exercise regardless of age.

Although the majority of research on acute exercise and cognition has focused on adult populations, a more recent focus has been on pediatric populations. Reviews of early behavioral studies testing this relationship suggest that school age children also may derive cognitive benefits from physical activity participation (Sibley and Etnier 2003; Tomporowski 2003a). Accordingly, Hillman et al. (2009b) assessed the extent to which improvements in cognition following a single acute bout of moderate intensity aerobic exercise effect both basic (i.e., laboratory tests) and applied (i.e., scholastic performance) aspects of cognition in preadolescent children. Findings from this investigation revealed that following a single $20 \mathrm{~min}$ bout of moderately intense treadmill walking, relative to seated rest, children exhibited improved response accuracy to a modified flanker task, had selectively larger P3 amplitudes only for incongruent trials, and performed better on the reading subtest of the Wide Range Achievement Test-3rd edition (Hillman et al. 2009b). Collectively, these findings indicate a positive effect of single acute bouts of aerobic exercise on inhibitory aspects of cognitive control, which appear to relate to facilitations in attentional resource allocation in an age- and exercisedependent manner, while cognitive processing and stimulus classification speed appears to more generally be influenced. Lastly, although evidence is sparse, preliminary results suggest that single acute bouts of exercise may serve to benefit scholastic performance, and thus may be an important consideration for educational practices.

\subsubsection{Action Monitoring}

Although the majority of previous investigations on acute exercise and neuroelectric indices of cognitive control have focused on the stimulus-locked P3 component, only a single study has investigated the effect of acute exercise on response-locked action monitoring processes as indexed by the ERN component. Themanson and Hillman (2006) examined action monitoring processes in response to a flanker task in a sample of college-aged young adults. Findings revealed no relationship between $30 \mathrm{~min}$ of moderate intensity aerobic exercise and neuroelectric indices of action monitoring (Themanson and Hillman 2006). Thus, these findings suggest that acute exerciseinduced changes in neuroelectric processes underlying cognitive control are selective to the allocation of attentional resources and appear to be unrelated to action monitoring processes. It is important to note, however, that this investigation only assessed college-aged young adults who collectively were performing the flanker task at an accuracy level above $90 \%$, which allowed for a relatively small number of error trials in which to observe modulation of the ERN. Thus, further research is necessary to better understand the effects of single acute bouts of exercise on action monitoring 

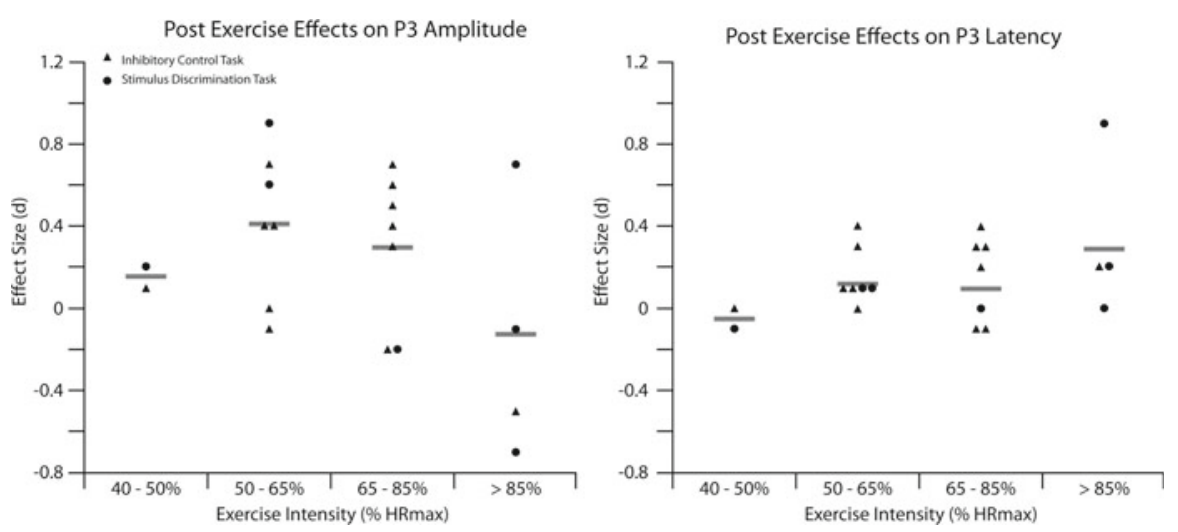

Fig. 18.4 Characterization of the influence of relative exercise intensity on P3 amplitude (left) and latency (right) following participation in a single bout of short duration aerobic physical activity. Points indicate individual effect sizes by cognitive task; bars indicate mean effect size for each exercise intensity range

processes under conditions allowing a greater number of errors to achieve a more robust index of the neuroelectric indices underlying action monitoring.

\subsubsection{Summary}

Collectively, findings from these investigations have suggested that a 20-30 min bout of aerobic exercise generally serves to increase the allocation of attentional resources (as indexed by larger amplitude of the P3 component), and facilitates cognitive processing and stimulus classification speed (as indexed by shorter latency of the P3 component) across a number of cognitive tasks. Further, as illustrated in Fig. 18.4, these exercise-induced enhancements in cognition appear to relate to the relative exercise intensity (in a curvilinear fashion). Based on visual inspection of the studies described herein (see Fig. 18.4), the greatest enhancements in the amplitude of the P3 component appear following exercise at an intensity between 50 and $65 \%$ of maximal heart rate; however, clear exceptions to this rule exist (see Hillman et al. 2003). Although it should be noted that this optimal intensity may be task dependent with more cognitively demanding workloads requiring more strenuous exercise intensity. Alternatively, enhancements in the latency of the P3 component appear to relate linearly to the intensity of the exercise bout.

\subsubsection{Neuroelectric Changes in Cognition During a Single Acute Bout of Physical Activity}

Although the research discussed previously has highlighted the beneficial effects of both chronic and acute physical activity participation on neuroelectric and behavioral 
indices of performance, it is important to note that a growing body of research suggests that transient decrements in cognition may occur during acute physical activity participation. Specifically, during moderately intense aerobic exercise, behavioral assessments have observed an impairment in perception (Paas and Adam 1991) and cognitive control (Dietrich and Sparling 2004). Alternatively, other researchers have observed a facilitation in performance on tasks of decision making (Arcelin et al. 1998; Davranche and Audiffren 2004; Paas and Adam 1991) and response conflict (Davranche and McMorris 2009). The application of neuroimaging techniques may provide additional insight into these conflicting results; however to date, relatively few investigations have assessed changes in the cognitive processes underlying these behavioral changes in performance during acute exercise. This, in part, may be due to the additional noise (artifact) that is associated with the gross motor movement inherent in physical activity behaviors. Recent technological advancements using "dry" electrodes and careful methodological techniques (see Pontifex and Hillman 2008 for further discussion), however, will allow future researchers greater access to apply these neuroimaging methods to assess changes in cognition during exercise. Thus far, only two investigations have assessed changes in neuroelectric indices of cognition during short duration aerobic exercise. Although both of these investigations have assessed the P3 component, they each assessed a different aspect of cognition.

\subsubsection{Stimulus Discrimination}

Yagi et al. (1999) assessed how stimulus discrimination processes may be modulated during $10 \mathrm{~min}$ of aerobic exercise at a workload equivalent of $65-75 \%$ of agepredicted maximum heart rate in a sample of 24 college-aged young adults. To reduce the potential influence of the gross motor movement of exercise, participants exercise on a recumbent cycle ergometer while completing auditory $(1,000 \mathrm{~Hz}$ nontarget tone and $2,000 \mathrm{~Hz}$ target tone) and visual (white " $\mathrm{X}$ " nontarget stimulus, white "O" target stimulus) oddball tasks. Findings from this investigation revealed that during exercise, participants exhibited smaller P3 amplitude and shorter P3 latency for both the auditory and visual oddball tasks, relative to pre-exercise measures (Yagi et al. 1999). Although some methodological confounds exist with regard to potential practice effects, these findings suggest that the capacity to allocate attentional resources may be reduced during aerobic exercise, but the processing of environmental information may be facilitated.

\subsubsection{Inhibition}

Pontifex and Hillman (2007) assessed the influence of moderately intense aerobic exercise on inhibitory control using a modified flanker task (Eriksen and Eriksen 
1974) in a sample of 41 college-aged young adults. Participants completed counterbalanced conditions of rest and $6.5 \mathrm{~min}$ of steady-state exercise on a cycle ergometer at $60 \%$ of their maximum heart rate. Findings from this investigation revealed an increase in P3 amplitude over bilateral frontal electrode sites as well as a global increase in P3 latency during exercise relative to rest. Further, Pontifex and Hillman (2007) assessed the influence of aerobic exercise on earlier ERP components. Specifically, the N1, P2, and N2 components were assessed, which relate to aspects of visual discrimination (Luck 1995; Vogel and Luck 2000), selective attention (Talsma and Kok 2001), and response inhibition (Ridderinkhof et al. 2002), respectively. During exercise, Pontifex and Hillman (2007) observed a parietal reduction in N1 amplitude, increased fronto-central P2 amplitude, and global reductions in N2 amplitude, relative to seated rest. Accordingly, these findings suggest that during aerobic exercise participants experienced a decreased ability to visually discriminate stimuli (N1 amplitude), which requires an increase in selective attention (P2 amplitude), and results in reductions in response conflict (N2 amplitude). These earlier neural inefficiencies thus necessitate a greater allocation of attentional resources toward stimulus engagement (P3 amplitude) and further relate to delays in stimulus evaluation and classification speed (P3 latency). As an extension of these deficits in stimulus acquisition and cognitive processing, participants exhibited behavioral deficits during exercise in response to the incongruent trials, which place greater demands on inhibitory control. Together, these data suggest that the neural inefficiencies that occur during exercise may only manifest as behavioral deficits when the cognitive load is high, with lower cognitive loads seemingly unaffected when measured via overt indices of task performance.

\subsubsection{Summary}

Given the small and disparate body of literature to date, which has examined modulations in neuroelectric indices of cognition that occur during exercise, it is difficult to make broad generalizations. However, these findings do provide preliminary evidence to suggest that the neural networks underlying aspects of cognition may be differentially influenced during physical activity participation. Thus, future investigations assessing this relationship must be particularly cognizant of the nature of the task to be utilized.

\subsection{Future Directions}

With few exceptions (Dustman et al. 1990; Polich and Lardon 1997), the vast majority of research on the relation of both chronic and acute exercise to neuroelectric indices of cognition has occurred during the last decade. As such, this field is in its infancy, and there are a number of directions that warrant future consideration. 
For instance, considerable investigation has pursued stimulus discrimination and inhibitory aspects of cognition, with only a sparse few studies examining other types of cognition. Memory processes are especially poorly understood at this time. Despite several strong early attempts to better understand the relation of exercise to memory, a programmatic approach to this area is needed. Similar arguments can be made for aspects of attention and cognitive control as well. As this area continues to build, the examination of the different ERP components is necessary to better determine which aspects of cognition are selectively influenced by exercise.

Of equal importance, all prior ERP research examining chronic exercise has employed cross-sectional designs. Although the cross-sectional evidence reported herein has, for the most part, been consonant in its findings, future research must employ more rigorous study designs to more definitively determine the causal effects of chronic exercise on neuroelectric indices of cognition. Recent research in the Neurocognitive Kinesiology Laboratory at the University of Illinois has pursued this question in preadolescent children. The Fitness Improves Thinking in Kids (FITKids) trial began in 2007 and remains ongoing at the time of this chapter. However, preliminary data from the first $120+$ participants suggest that daily participation in moderate to vigorous physical activity relates to larger P3 amplitude and shorter P3 latency across a number of cognitive tasks that tap attention, inhibition, and stimulus discrimination. Clearly, other randomized control trials are needed to expound the exercise and cognition database to provide a clear and more definitive understanding of the benefits of exercise to brain health and cognition.

Relative to the acute, transient effects of exercise on cognition, more research is, quite simply, necessary. A review of the available literature on this topic has evidenced conflicting results, but also a number of methodological shortcomings, which limit our understanding not only of the transient effects of acute exercise on cognition, but also of the basis for the conflicting findings. Further, future research needs to programmatically examine exercise mode, intensity, and duration to determine the nature of the "dose-response" relationship between single bouts of exercise and cognition. Along these same lines, programmatic study of the enduring effects of exercise on cognition is needed. Based on a cursory examination of the duration of the effects observed stemming from a single acute bout of exercise, it appears that alterations in cognitive function last approximately one hour from the cessation of the acute exercise bout (Hillman et al. 2003, 2009b). More definitive exploration of the nature and timing of these cognitive changes are sorely needed, as they may have a direct application to cognitive performance in schools and the workplace.

Finally, future exercise-ERP research should begin to bridge basic laboratory science with "real-world" settings in which exercise and cognitive performance occur. That is, ERPs reflect externally valid cognitive operations, and simply understanding the nature of the relationship between exercise and ERPs falls short of understanding how exercise may influence cognition during daily life. Determining the relationship between exercise, ERPs, and cognition in daily life may serve to better inform scientists on the selective aspects of cognition that are affected by exercise and increase the cognitive health and effective functioning of individuals as they progress through the lifespan. 


\subsection{Conclusions}

In summary, the body of research described herein details the relation of chronic and acute exercise participation on neuroelectric indices of brain health and cognition. A growing body of research amassed over the last decade has suggested that chronic participation in physical activity and higher amounts of aerobic fitness are related to better cognitive performance across a variety of cognitive tasks, with disproportionately larger benefits observed for tasks necessitating greater amounts of cognitive control. Similarly, single bouts of acute exercise also positively affect cognition and its neuroelectric underpinnings, with superior performance noted during the time following the cessation of exercise. However, a very small body of research suggests that similar benefits are not accrued for attentional tasks during the exercise bout. Accordingly, future research is warranted to not only detail the aspects of exercise and cognition that lead to positive health outcomes, but also the limitations of this relationship. Regardless, ERPs have provided a unique tool for understanding the select aspects of cognition, which occur between stimulus engagement and response production that are altered by exercise. The overall goal of this body of research is to maximize brain health and cognition, leading to increased effective functioning of individuals during their daily lives.

\section{References}

Arcelin R, Delignieres D, Brisswalter J (1998) Selective effects of physical exercise on choice reaction processes. Percept Mot Skills 87:175-185

Barkley RA (1997) Behavioral inhibition, sustained attention, and executive functions. Psychol Bull 121:65-94

Bojko A, Kramer AF, Peterson MS (2004) Age equivalence in switch costs for prosaccade and antisaccade tasks. Psychol Aging 19:226-234

Booth FW, Lees SJ (2006) Physically active subjects should be the control group. Med Sci Sports Exerc 38:405-406

Bortz WM II (1985) Physical exercise as an evolutionary force. J Hum Evol 14:145-155

Botvinick MM, Braver TS, Barch DM, Carter CS, Cohen JD (2001) Conflict monitoring and cognitive control. Psychol Rev 108:624-652

Brisswalter J, Collardeau M, Arcelin R (2002) Effects of acute physical exercise on cognitive performance. Sports Med 32:555-566

Brunia CH, van Boxtel GJ (2001) Wait and see. Int J Psychophysiol 43:59-75

Bunge SA, Crone EA (2009) Neural correlates of the development of cognitive control. In: Rumsey JM, Ernst M (eds) Neuroimaging in developmental clinical neuroscience. Cambridge University Press, New York, NY

Carrier DR (1984) The energetic paradox of human running and hominid evolution. Curr Anthropol 25:483-495

Carter CS, Braver TS, Barch DM, Botvinick MM, Noll D, Cohen JD (1998) Anterior cingulated cortex, error detection, and the online monitoring of performance. Science 280:747-749

Carter CS, van Veen V (2007) Anterior cingulate cortex and conflict detection: an update of theory and data. Cogn Affect Behav Neurosci 7:367-379

Caterino MC, Polack ED (1999) Effects of two types of activity on the performance of second-, third-, and fourth-grade students on a test of concentration. Percept Mot Skills 89:245-248 
Center for Disease Control and Prevention (2008) Prevalence of self-reported physically active adults - United States, 2007. MMWR Morb Mortal Wkly Rep 57:1297-1300

Colcombe SJ, Kramer AF (2003) Fitness effects on the cognitive function of older adults: a metaanalytic study. Psychol Sci 14:125-130

Colcombe SJ, Kramer AF, Erickson KI, Scalf P, McAuley E, Cohen NJ, Webb A, Jerome GJ, Marquez DX, Elavsky S (2004) Cardiovascular fitness, cortical plasticity, and aging. Proc Natl Acad Sci USA 101:3316-3321

Coles MGH, Gratton G, Fabiani M (1990) Event-related potentials. In: Cacioppo JT, Tassinary LG (eds) Principles of psychophysiology: physical, social, and inferential elements. Cambridge University Press, New York, NY, pp 413-455

Cui RQ, Egkher A, Huter D, Lang W, Lindinger G, Deecke L (2000) High resolution spatiotemporal analysis of the contingent negative variation in simple or complex motor tasks and a nonmotor task. Clin Neurophysiol 111:1847-1859

Davidson MC, Amso D, Anderson LC, Diamond A (2006) Development of cognitive control and executive functions from 4 to 13 years: evidence from manipulations of memory, inhibition, and task switching. Neuropsychologia 44:2037-2078

Davranche K, Audiffren M (2004) Facilitating effects of exercise on information processing. J Sports Sci 22:419-428

Davranche K, McMorris T (2009) Specific effects of acute moderate exercise on cognitive control. Brain Cogn 69:565-570

Dehaene S, Posner MI, Tucker DM (1994) Localization of a neural system for error detection and compensation. Psychol Sci 5:303-305

Department of Health and Human Services [DHHS] and Department of Education [DOE] (2000) Promoting better health for young people through physical activity and sports. A Report to the President from the Secretary of Health and Human Services and the Secretary of Education. Centers for Disease Control, Silver Spring, MD

Diamond A (2006) The early development of executive functions. In: Bialystok E, Craik FIM (eds) Lifespan cognition: mechanism of change. Oxford University Press, New York, NY, pp 70-95

Dietrich A, Sparling PB (2004) Endurance exercise selectively impairs prefrontal-dependent cognition. Brain Cogn 55:516-524

Donchin E (1981) Surprise!...Surprise? Psychophysiology 18:493-513

Dustman RE, Emmerson RY, Ruhling RO, Shearer DE, Steinhaus LA, Johnson SC, Bonekat HW, Shigeoka JW (1990) Age and fitness effects on EEG, ERPs, visual sensitivity, and cognition. Neurobiol Aging 11:193-200

Duzova H, Özi ik HI, Polat A, Emre MH, Gullu E (2005) Correlations between event-related potential components and nitric oxide in maximal anaerobic exercise among sportsmen trained at various levels. Int J Neurosci 115:1353-1373

Ebmeier KP, Steele JD, MacKenzie DM, O’Carroll RE, Kydd RR et al (1995) Cognitive brain potentials and regional cerebral blood flow equivalents during two- and three-sound auditory "oddball tasks". Clin Neurophysiol 95:434-443

Ellemberg D, St-Louis-Deschênes M (2010) The effect of acute physical exercise on cognitive function during development. Psychol Sport Exerc 11:122-126

Emmerson RY, Dustman RE, Shearer DE, Turner CW (1989) P3 latency and symbol digit performance correlations in aging. Exp Aging Res 15:151-159

Eriksen CW, Eriksen BA (1974) Effects of noise letters upon the identification of a target letter in a non-search task. Percept Psychophys 16:143-149

Falkenstein M, Hohnsbein J, Hoormann J, Blanke L (1991) Effects of crossmodal divided attention on late ERP components: II. Error processing in choice reaction tasks. Clin Neurophysiol 78:447-455

Falkenstein M, Hoormann J, Hohnsbein J, Kleinsorge T (2003) Short-term mobilization of processing resources is revealed in the event-related potential. Psychophysiology 40:914-923

Fialkowski KR (1986) A mechanism for the origin of the human brain: a hypothesis. Curr Anthropol 27:288-290

Fontaine KR, Redden DT, Wang C, Westfall AO, Allison DB (2003) Years of life lost due to obesity. J Am Med Assoc 289:187-193 
Friedman D, Nessler D, Cycowicz YM, Horton C (2009) Development of and change in cognitive control: a comparison of children, young adults, and older adults. Cogn Affect Behav Neurosci 9:91-102

Gabbard C, Barton J (1979) Effects of physical activity on mathematical computation among young children. J Psychol 103:287-288

Gehring WJ, Goss B, Coles MGH, Meyer DE, Donchin E (1993) A neural system for error detection and compensation. Psychol Sci 4:385-390

Gehring WJ, Knight RT (2000) Prefrontal-cingulate interactions in action monitoring. Nat Neurosci 3:516-520

Gómez CM, Marco J, Grau C (2003) Preparatory visuo-motor cortical network of the contingent negative variation estimated by current density. Neuroimage 20:216-224

Hamano T, Luders HO, Ikeda A, Collura TF, Comair YG, Shibasaki H (1997) The cortical generators of the contingent negative variation in humans: a study with subdural electrodes. Electroencephalogr Clin Neurophysiol 104:257-268

Haskell WL, Lee IM, Pate RR, Powell KE, Blair SN, Franklin BA, Macera CA, Heath GW, Thompson PD, Bauman A (2007) Physical activity and public health: updated recommendation for adults from the American College of Sports Medicine and the American Heart Association. Med Sci Sports Exerc 39:1423-1434

Hillman CH, Belopolsky AV, Snook EM, Kramer AF, McAuley E (2004) Physical activity and executive control: implications for increased cognitive health during older adulthood. Res Q Exerc Sport 75:176-185

Hillman CH, Buck SM, Themanson JT, Pontifex MB, Castelli DM (2009a) Aerobic fitness and cognitive development: event-related brain potential and task performance indices of executive control in preadolescent children. Dev Psychol 45:114-129

Hillman CH, Castelli DM, Buck SM (2005) Aerobic fitness and neurocognitive function in healthy preadolescent children. Med Sci Sports Exerc 37:1967-1974

Hillman CH, Erickson KI, Kramer AF (2008) Be smart, exercise your heart: exercise effects on brain and cognition. Nat Rev Neurosci 9:58-65

Hillman CH, Kramer AF, Belopolsky AV, Smith DP (2006) A cross-sectional examination of age and physical activity on performance and event-related brain potentials in a task switching paradigm. Int J Psychophysiol 59:30-39

Hillman CH, Pontifex MB, Raine LB, Castelli DM, Hall EE, Kramer AF (2009b) The Effect of acute treadmill walking on cognitive control and academic achievement in preadolescent children. Neuroscience 159:1044-1054

Hillman CH, Snook EM, Jerome GJ (2003) Acute cardiovascular exercise and executive control function. Int J Psychophysiol 48:307-314

Howard L, Polich J (1985) P300 latency and memory span development. Dev Psychol 21:283-289

Hruby T, Marsalek P (2003) Event-related potentials - the P3 wave. Acta Neurobiol Exp 63:55-63

Hugdahl K (1995) Psychophysiology: the mind-body perspective. Harvard University Press, Cambridge, MA

Ilan AB, Polich J (1999) P300 and response time from a manual stroop task. Clin Neurophysiol 110:367-373

Johnson R Jr, Pfefferbaum A, Kopell BS (1985) P300 and long-term memory: latency predicts recognition performance. Psychophysiology 22:497-507

Kamijo K, Hayashi Y, Sakai T, Yahiro T, Tanaka K, Nishihira Y (2009) Acute effects of aerobic exercise on cognitive function in older adults. J Gerontol 64:356-363

Kamijo K, Nishihira Y, Hatta A, Kaneda T, Wasaka T, Kida T, Kuroiwa K (2004) Differential influences of exercise intensity on information processing in the central nervous system. Eur $\mathrm{J}$ Appl Physiol 92:305-311

Kamijo K, Nishihira Y, Higashiura T, Kuroiwa K (2007) The interactive effect of exercise intensity and task difficulty on human cognitive processing. Int J Psychophysiol 65:114-121

Kamijo K, O'Leary KC, Pontifex MB, Themanson JR, Hillman CH (2010) The relation of aerobic fitness to neuroelectric indices of cognitive and motor task preparation. Psychophysiology $47: 814-821$ 
Kamijo K, Pontifex MB, O’Leary KC, Scudder MR, Wu CT, Castelli DM, Hillman CH (2011) The effects of an afterschool physical activity program on working memory in preadolescent children. Dev Sci 14:1046-1058

Kamijo K, Takeda Y (2009) General physical activity levels influence positive and negative priming effects in young adults. Clin Neurophysiol 120:511-519

Kamijo K, Takeda Y (2010) Regular physical activity improves executive function during task switching in young adults. Int J Psychophysiol 75:304-311

Kirino E, Belger A, Goldman-Rakic P, McCarthy G (2000) Prefrontal activation evoked by infrequent target and novel stimuli in a visual target detection task: an event-related functional magnetic resonance imaging study. J Neurosci 20:6612-6618

Kramer AF, Hahn S, Cohen NJ, Banich MT, McAuley E, Harrison CR, Chason J, Vakil E, Bardell L, Bolleau RA, Colcombe A (1999) Ageing, fitness and neurocognitive function. Nature 400:416-419

Kray J, Lindenberger U (2000) Adult age differences in task switching. Psychol Aging 15:126-147

Lambourne K, Tomporowski P (2010) The effect of exercise-induced arousal on cognitive task performance: a meta-regression analysis. Brain Res 1341:12-24

Leynes PA, Allen JD, Marsh RL (1998) Topographic differences in CNV amplitude reflect different preparatory processes. Int J Psychophysiol 31:33-44

Lorist MM, Klein M, Nieuwenhuis S, De Jong R, Mulder G, Meijman TF (2000) Mental fatigue and task control: planning and preparation. Psychophysiology 37:614-625

Loveless NE, Sanford AJ (1974) Slow potential correlates of preparatory set. Biol Psychol $1: 303-314$

Luck SJ (1995) Multiple mechanisms of visual-spatial attention: recent evidence from human electrophysiology. Behav Brain Res 71:113-123

Magliero A, Bashore TR, Coles MGH, Donchin E (1984) On the dependence of P300 latency on stimulus evaluation processes. Psychophysiology 21:171-186

Magnié MN, Bermon S, Martin F, Madany-Lounis M, Suisse G, Muhammad W, Dolisi C (2000) P300, N400, aerobic fitness and maximal aerobic exercise. Psychophysiology 37:369-377

Mahar MT, Murphy SK, Rowe DA, Golden J, Shields AT, Raedeke TD (2006) Effects of a classroom-based program on physical activity and on-task behavior. Med Sci Sports Exerc 38:2086-2094

McCarthy G, Donchin E (1981) A metric for thought: a comparison of P300 latency and reaction time. Science 211:77-80

McNaughten D, Gabbard C (1993) Physical exertion and the immediate mental performance of sixth-grade children. Percept Mot Skills 77:1155-1159

Meyer DE, Kieras DE (1997) A computational theory of executive cognitive processes and multitask performance: Part 1. Basic mechanisms. Psychol Rev 104:3-65

Miltner WHR, Lemke U, Weiss T, Holroyd C, Scheffers MK, Coles MGH (2003) Implementation of error-processing in the human anterior cingulated cortex: a source analysis of the magnetic equivalent of the error-related negativity. Biol Psychol 64:157-166

Miyake A, Friedman NP, Emerson MJ, Witzki AH, Howerter A (2000) The unity and diversity of executive functions and their contributions to complex "frontal lobe" tasks: a latent variable analysis. Cogn Psychol 41:49-100

Nakamura Y, Nishimoto K, Akamatu M, Takahashi M, Maruyama A (1999) The effect of jogging on the P300 event related potentials. Clin Neurophysiol 39:71-74

Nieuwenhuis S, Ridderinkhof KR, Blom J, Band GPH, Kok A (2001) Error-related brain potentials are differentially related to awareness of response errors: evidence from an antisaccade task. Psychophysiology 38:752-760

Norman DA, Shallice T (1986) Attention to action: willed and automatic control of behavior. In: Davidson RJ, Schwartz GE, Shapiro D (eds) Consciousness and self-regulation, vol 4, Advances in research and theory. Plenum, New York, NY, pp 1-18

Olshansky SJ, Passaro DJ, Hershow RC, Layden J, Carnes BA et al (2005) A potential decline in life expectancy in the United States in the 21st century. N Engl J Med 352:1138-1145 
Paas FGWC, Adam JJ (1991) Human information processing during physical exercise. Ergonomics 34:1385-1397

Polich J (1987) Task difficulty, probability and inter-stimulus interval as determinants of P300 from auditory stimuli. Clin Neurophysiol 63:251-259

Polich J (2003) Overview of P3a and P3b. In: Polich J (ed) Detection of change: event-related potential and fMRI findings. Kluwer, Boston, pp 83-98

Polich J (2007) Updating P300: an integrative theory of P3a and P3b. Clin Neurophysiol 118:2128-2148

Polich J, Kok A (1995) Cognitive and biological determinants of P300: an integrative review. Biol Psychol 41:103-146

Polich J, Martin S (1992) P300, cognitive capability, and personality. Pers Individ Dif 13:533-543

Polich J, Howard L, Starr A (1983) P300 latency correlates with digit span. Psychophysiology 20:665-669

Polich J, Lardon MT (1997) P300 and long-term physical exercise. Clin Neurophysiol 103:493-498

Pontifex MB, Hillman CH (2007) Neuroelectric and behavioral indices of interference control during acute cycling. Clin Neurophysiol 118:570-580

Pontifex MB, Hillman CH (2008) Neuroelectric measurement of cognition during aerobic exercise. Methods 45:271-278

Pontifex MB, Hillman CH, Fernhall B, Thompson KM, Valentini TA (2009a) The effect of acute aerobic and resistance exercise on working memory. Med Sci Sports Exerc 41:927-934

Pontifex MB, Hillman CH, Polich JP (2009b) Age physical fitness, and attention: P3a P3b. Psychophysiology 46:379-387

Pontifex MB, Raine LB, Johnson CR, Chaddock L, VanPatter M, Voss MW et al (2011) Cardiorespiratory fitness and the flexible modulation of cognitive control in preadolescent children. J Cogn Neurosci 23:1332-1345

Ridderinkhof KR, de Vulgt Y, Bramlage A, Spaan M, Elton M, Snel J, Band GPH (2002) Alcohol consumption impairs detection of performance errors in mediofrontal cortex. Science 298:2209-2211

Rogers RD, Monsell S (1995) Costs of a predictable switch between simple cognitive tasks. J Exp Psychol 124:207-231

Scisco JL, Leynes PA, Kang J (2008) Cardiovascular fitness and executive control during taskswitching: an ERP study. Int J Psychophysiol 69:52-60

Sibley BA, Etnier JL (2003) The relationship between physical activity and cognition in children: a meta-analysis. Pediatr Exerc Sci 15:243-256

Smith PJ, Blumenthal JA, Hoffman BM, Cooper H, Strauman TA, Welsh-Bohmer K, Browndyke JN, Sherwood A (2010) Aerobic exercise and neurocognitive performance: a meta-analytic review of randomized controlled trials. Psychosom Med 72:239-252

Strong WB, Malina RM, Blimke CJR, Daniels SR, Dishman RK, Gutin B, Hergenroeder AC et al (2005) Evidence based physical activity for school-age youth. J Pediatr 146:732-737

Stroth S, Kubesch S, Dieterle K, Ruchsow M, Heim R, Kiefer M (2009) Physical fitness, but not acute exercise modulates event-related potential indices for executive control in healthy adolescents. Brain Res 1269:114-124

Sutton S, Braren M, Zubin J, John ER (1965) Evoked-potential correlates of stimulus uncertainty. Science 150:1187-1188

Talsma D, Kok A (2001) Nonspatial intermodal selective attention is mediated by sensory brain areas: evidence from event-related potentials. Psychophysiology 38:736-751

Themanson JR, Hillman CH (2006) Cardiorespiratory fitness and acute aerobic exercise effects on neuroelectric and behavioral measures of action monitoring. Neuroscience 141:757-767

Themanson JR, Hillman CH, Curtin JJ (2006) Age and physical activity influences on action monitoring during task switching. Neurobiol Aging 27:1335-1345

Themanson JR, Pontifex MB, Hillman CH (2008) Fitness and action monitoring: evidence for improved cognitive flexibility in young adults. Neuroscience 157:319-328

Tipper SP, Brehaut JC, Driver J (1990) Selection of moving and static objects for the control of spatially directed action. J Exp Psychol Hum Percept Perform 16:492-504 
Tomporowski PD (2003a) Cognitive and behavioral responses to acute exercise in youths: a Review. Pediatr Exerc Sci 15:348-359

Tomporowski PD (2003b) Effects of acute bouts of exercise on cognition. Acta Psychol 112:297-324

van Boxtel GJ, Brunia CH (1994) Motor and non-motor aspects of slow brain potentials. Biol Psychol 38:37-51

van Veen V, Carter CS (2002) The timing of action-monitoring processes in the anterior cingulated cortex. J Cogn Neurosci 14:593-602

Vaynman S, Gomez-Pinilla F (2006) Revenge of the "sit": how lifestyle impacts neuronal and cognitive health though molecular systems that interface energy metabolism with neuronal plasticity. J Neurosci Res 84:699-715

Verleger R (1997) On the utility of P3 latency as an index of mental chronometry. Psychophysiology 34:131-156

Vogel EK, Luck SJ (2000) The visual N1 component as an index of a discrimination process. Psychophysiology 37:190-203

Weerts TC, Lang PJ (1973) The effects of eye fixation and stimulus and response location on the contingent negative variation (CNV). Biol Psychol 1:1-19

Wild-Wall N, Hohnsbein J, Falkenstein M (2007) Effects of ageing on cognitive task preparation as reflected by event-related potentials. Clin Neurophysiol 118:558-569

Yagi Y, Coburn KL, Estes KM, Arruda JE (1999) Effects of aerobic exercise and gender on visual and auditory P300, reaction time, and accuracy. Eur J Appl Physiol 80:402-408

Yeung N, Botvinick MM, Cohen JD (2004) The neural basis of error detection: conflict monitoring and the error-related negativity. Psychol Rev 111:931-959 